\title{
A VIRTUAL EQUIPMENT AS A TEST BENCH FOR EVALUATING VIRTUAL METROLOGY ALGORITHMS
}

\author{
Andreas Mattes \\ Matthias Koitzsch \\ Dirk Lewke \\ Michael Müller-Zell \\ Martin Schellenberger \\ Fraunhofer Institute of Integrated Systems and Device Technology (IISB) \\ Schottkystr. 10 \\ 91058 Erlangen, GERMANY
}

\begin{abstract}
This paper presents a Virtual Equipment which serves as a testing environment for evaluating Virtual Metrology (VM) algorithms prior to their implementation into semiconductor fab structures. The Virtual Equipment merges statistical simulation with physical simulation to generate test data sets for various common and uncommon states of the processing equipment. The input data is based on historical fab data and synthetically generated data. Main result of the presented work is the bidirectional link of statistical methods with physical simulations which is the core of the virtual test environment. The testing of VM algorithms can be controlled via a Graphical User Interface (GUI). A simplified physical simulation of a Chemical Vapor Deposition (CVD) reaction chamber is set up based on CAD data as an example of the physical simulation part.
\end{abstract}

\section{INTRODUCTION AND MOTIVATION}

Challenges driven by Moore's Law (Moore 1965) increase the need for higher reliability in equipment and process control, e.g. for wafer to wafer and even within wafer control loops. The same is true for so called More-Than-Moore applications with increasing challenges of system integration. A promising approach for enhanced process control is applying Virtual Metrology (VM) techniques. VM is a concept to predict post process physical and electrical quality parameters of wafers and/or devices based on information collected from the manufacturing tools, e.g., equipment or process parameters. That includes support from other available sources like production context information or up-stream metrology. Quality parameters are, for example, layer thickness, electrical properties and critical dimensions. As a result VM increases process control at wafer to wafer level without measuring each wafer with standard metrology techniques. Up to now, VM algorithms are being researched and developed (e.g., Chang et al. 2006, Hung et al. 2007), yet they are still far from being state of the art at Integrated Device Manufacturers' (IDM) factories. To ensure optimal matching and accuracy of a specific VM technique in an existing fabrication line, VM algorithms have to be comprehensively tested and assessed prior to implementation. That requires a flexible testing environment for evaluating VM algorithms. The following sections describe in detail the test bench approach for testing VM algorithms. Standard statistical methods are taken into account as well as equipment simulations of a CVD reaction chamber. A Graphical User Interface based on Matlab/Simulink is illustrated. 


\section{DESCRIPTION OF THE TEST BENCH APPROACH - VIRTUAL EQUIPMENT}

Core aspect of the test bench is a virtual manufacturing equipment that simulates real equipment behavior and which is capable of simulating scenarios differing from the original fab data. The general approach and the concept of the Virtual Equipment are illustrated in Figure 1.

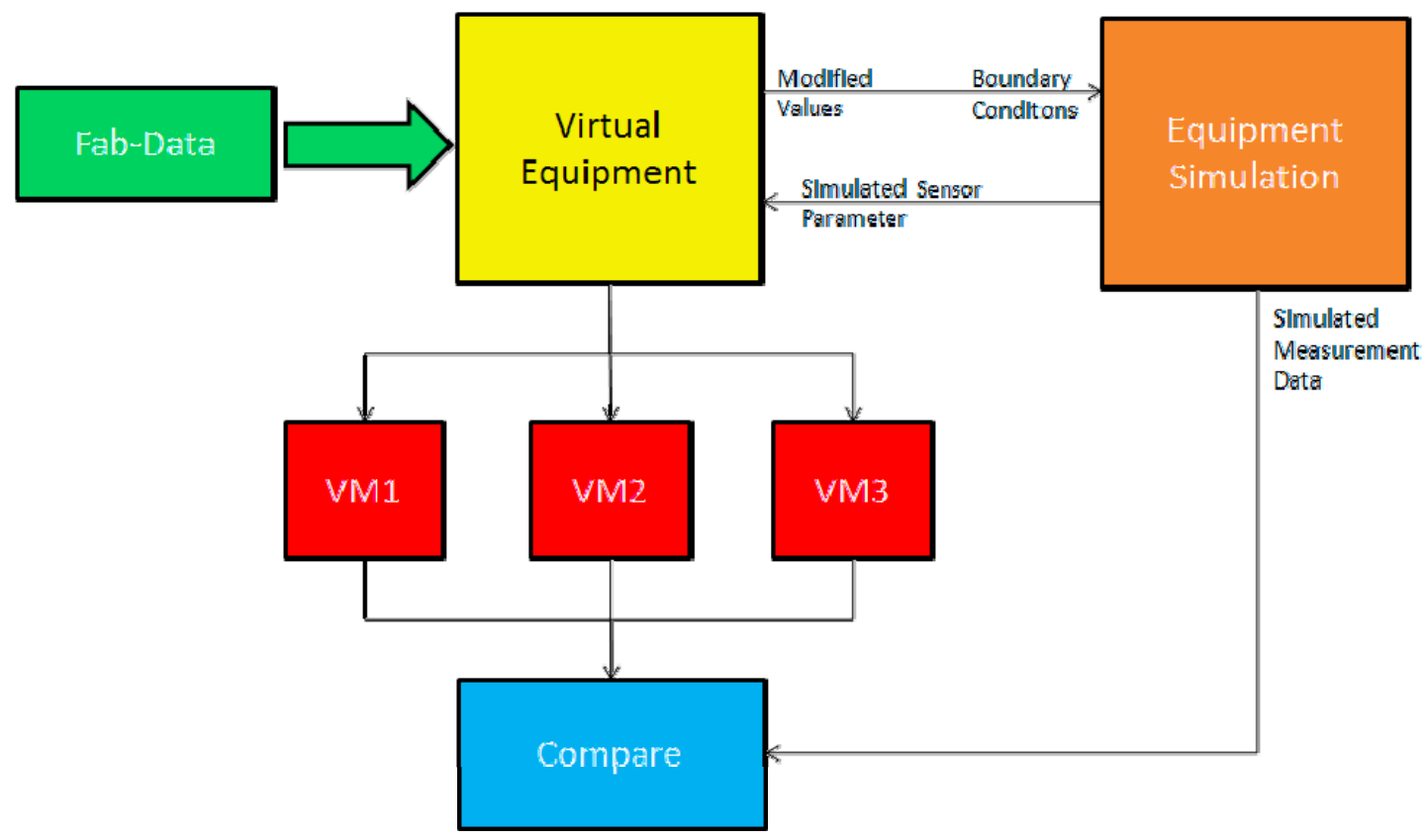

Figure 1: Concept of the Virtual Equipment; colors highlight the different functional components, which are described in the text

A mixture of statistical and physical simulation techniques is used to test different VM algorithms and to compare their gage capability both at standard conditions and faulty equipment states. VM algorithms can be selected and plugged in the Virtual Equipment for evaluation and comparison.

Main input for the Virtual Equipment is historical fab data provided by IDM project partners (green part in Figure 1). The Virtual Equipment modifies the fab data which serves as input for VM algorithms. Based on the input data from the Virtual Equipment, VM algorithms predict quality parameters of a particular process result (red part in Figure 1). With the current state of the Virtual Equipment, three different kinds of input data are possible:

- The historical fab data is transferred directly to the selected VM algorithms without any modifications. That is done to compare VM predicted process results with measured process results on the original fab scenarios.

- Statistical methods are applied either to the historical fab data or to synthetically generated data to simulate relevant equipment and process behavior, e.g., process drift, noise and maintenance actions (yellow part in Figure 1). That is done to explore the behavior of the individual VM algorithms on specific scenarios, especially faulty equipment states differing from the normal behavior which makes up most of the input data.

- Using a part of the signals in the altered historical fab data as boundary conditions, physical equipment simulations are used to compute virtual sensor output data (orange part in Figure 1). That provides more realistic fitting modifications for the rest of the signals than mere statistical methods. The major gain from that method is that you also get an estimation of quality parameters which can be used to judge the gage capability of VM algorithms in the simulated scenarios. 
For the research presented in this paper we focused on polysilicon deposition in a Rapid Thermal Processing (RTP) reactor as an exemplary physical simulation.

Predicted results from VM algorithms and simulated process results from equipment simulation are visualized. They are compared with each other to assess the behavior of VM algorithms dependent on various input scenarios (blue part in Figure 1).

The implementation of the GUI and the statistical simulation is done in Matlab/Simulink while the physical simulation uses CFD-ACE. Figure 2 shows the main Simulink model of the Virtual Equipment. The colors correspond to Figure 1. Model simulation is started from the GUI after the parameters are set. Physical simulation is contained in the RTPSIM block.

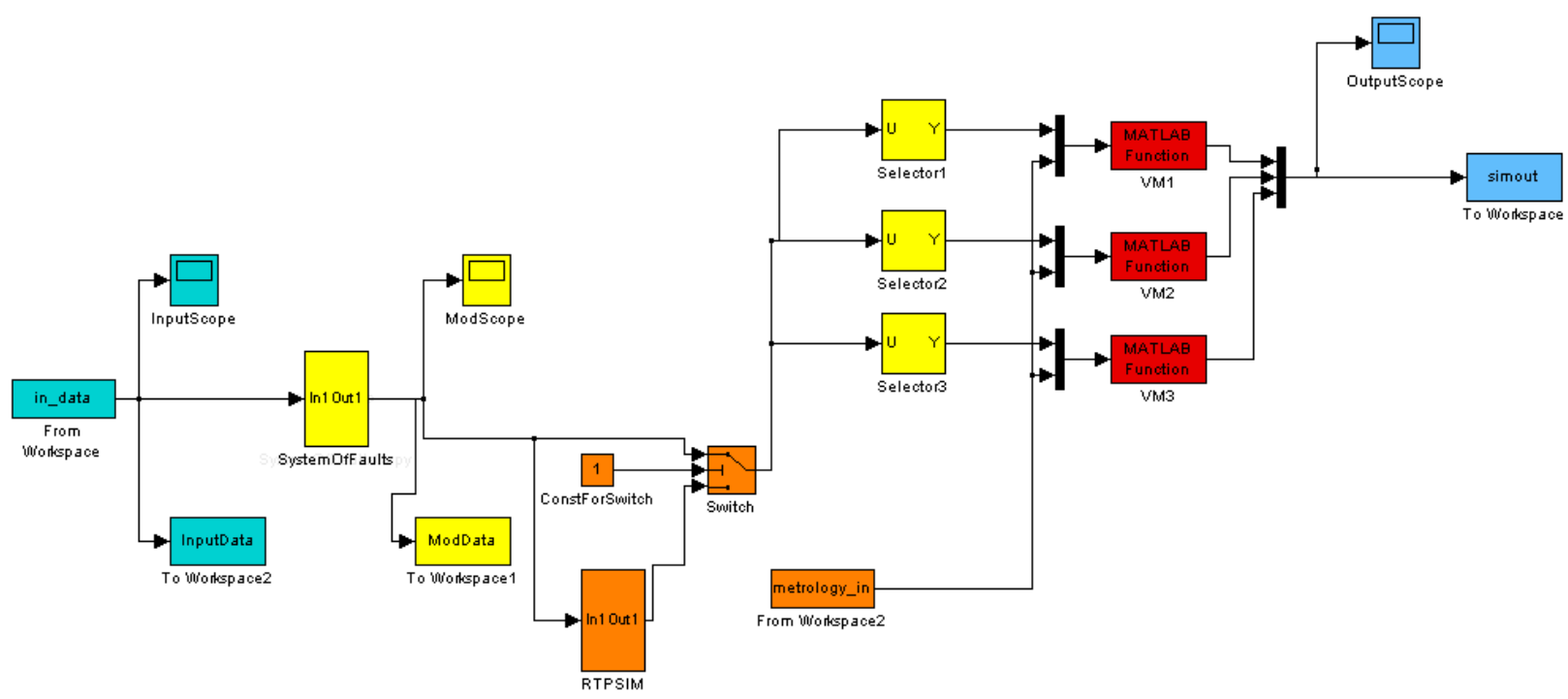

Figure 2: Matlab/Simulink model of the Virtual Equipment; colors correspond to Figure 1

\subsection{Statistical Methods}

The statistical modules are implemented as blocks in the main Simulink model of the Virtual Equipment (see SystemOfFaults block in Figure 2). Various modules using statistical methods have been added to simulate relevant process and equipment behavior and to generate new test data from historical fab data. Some of the modules simulate faults in sensors that are not part of a control loop. That only changes the values that the VM observes but not the process result itself and the metrology results in the original data can be used directly. Other changes require that the fault scenario is made in a consistent way or at least the results are interpreted by an expert.

Currently implemented methods are:

- Process drifts and offsets: Process variables often change from run-to-run in a linear way. This module allows for increasing or decreasing values continuously with time.

- Noise: The fab history data is already noisy but with this module a specific amount of extra noise can be added to the data. This can be interpreted as sensor noise or process result noise.

- Maintenance actions: Maintenance often changes certain signals in a non-continuous way. This module allows the addition of a specified signal (e.g., sawtooth) to parts of the data interpreted as changes due to maintenance actions.

- Outliers: Due to sensor or process faults single process runs might show largely differing values in one or more signals. This module adds outliers to the data. 
- Sensor deviation: If a sensor is not calibrated well, it can return values which are a certain percentage higher or lower than the measurement results should have been. This module models this behavior.

- Signal with same distribution: This module replaces a signal with a random signal with the same statistical distribution.

\subsection{Physical Simulation}

The current statistical methods in the Virtual Equipment can generate good test data but they require that the modules are used in a way that keeps the meaning and correlation between the different signals and the user has to estimate what the actual result of the VMs should have been with those changes. As improvement, physical simulation is added to the Virtual Equipment. Part of the fab data is changed with the statistical methods and then added as boundary conditions to the physical equipment model. As an exemplary equipment an RTP reactor is used and modeled within the multiphysics software CFD-ACE. Based on a simplified 2D-CAD-model of the equipment, flow, radiation, diffusion and chemistry are simulated. The values given are examples which are replaced by input from the Matlab/Simulink model in an actual run. Other Volume and Boundary Conditions required for the simulation are taken from Kim and Cho (2002), Fair (1993) and ESI-Group (2006).

The basis of the 2D-CAD-model is a 3D-CAD simulation model based on a real RTP reactor. Simulation results of the 3D-model were compared with process results of a real RTP reactor (Lewke 2011). The geometry was reduced from $3 \mathrm{D}$ to $2 \mathrm{D}$ due to computing time constraints of the test environment.

The simulated RTP reactor is a cold wall chamber with a quartz window on top to separate the chamber from the tungsten halogen lamps which are the heating source of the reactor. The wafer sits on a silicon carbide ( $\mathrm{SiC})$ ring. Process gas inlets and outlets are at the left and right side of the chamber, respectively, see Figure 3.

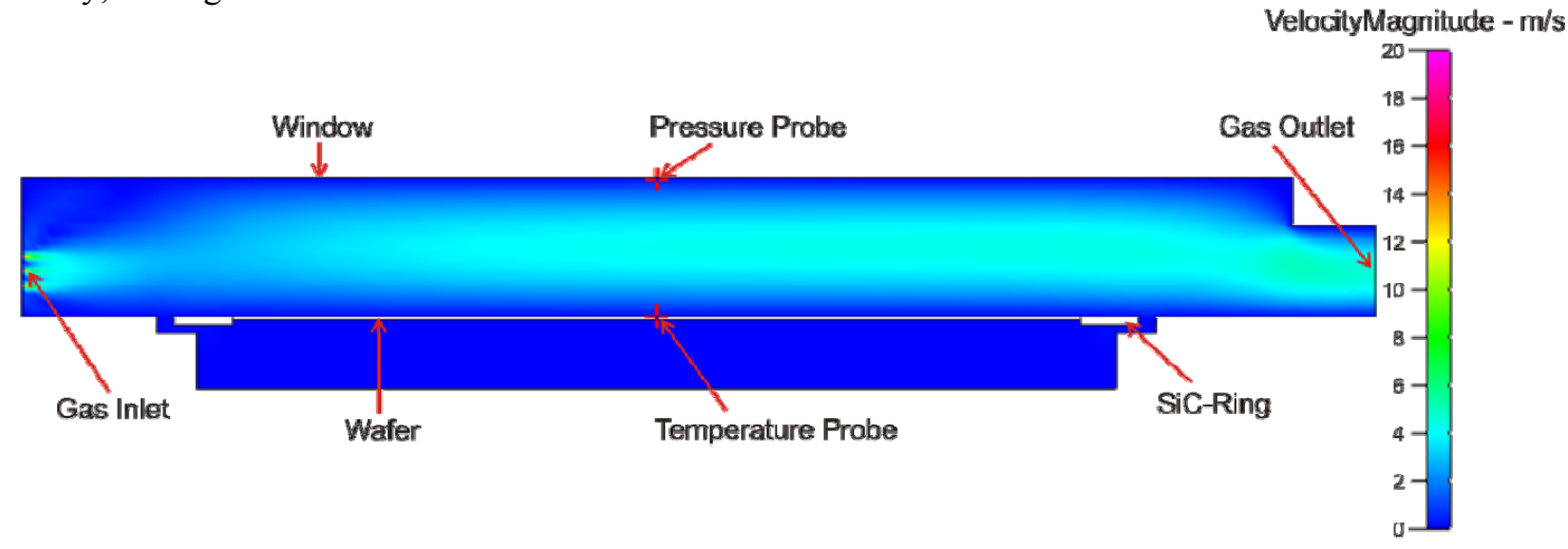

Figure 3: Simulation model of the RTP-reactor with colored velocity magnitude of the reactive gas. The positions of the virtual sensors are highlighted with red crosses

The quartz window is on the top of the model and is assumed to be the radiant heat source. To achieve a uniform temperature distribution over the wafer surface the top of the chamber is subdivided into twelve individually adjustable zones. The radiation temperature distribution all over the quartz window is set in the range of $1000 \mathrm{~K}$ to $1075 \mathrm{~K}$ to achieve a homogenous temperature on the wafer as possible.

The inlet consists of three holes with a diameter of $0.5 \mathrm{~mm}$. For the polysilicon deposition a mixture of argon (Ar) and silane $\left(\mathrm{SiH}_{4}\right)$ is used with mass fractions of $0.96 \%$ and $0.04 \%$ respectively. The surface reaction of polysilicon deposition from silane is: $\mathrm{SiH}_{4} \rightarrow \mathrm{Si}+2 \mathrm{H}_{2}$.

Relevant simulation results are saved in the model file, e.g., the chamber pressure, the temperature at the wafer top surface and the deposition rate. Virtual sensors for chamber pressure and wafer temperature 
were realized as point probes in the simulation model as can be seen in 4. Additional data for the temperature distribution, deposition rate, and pressure on the top of the wafer are also registered.

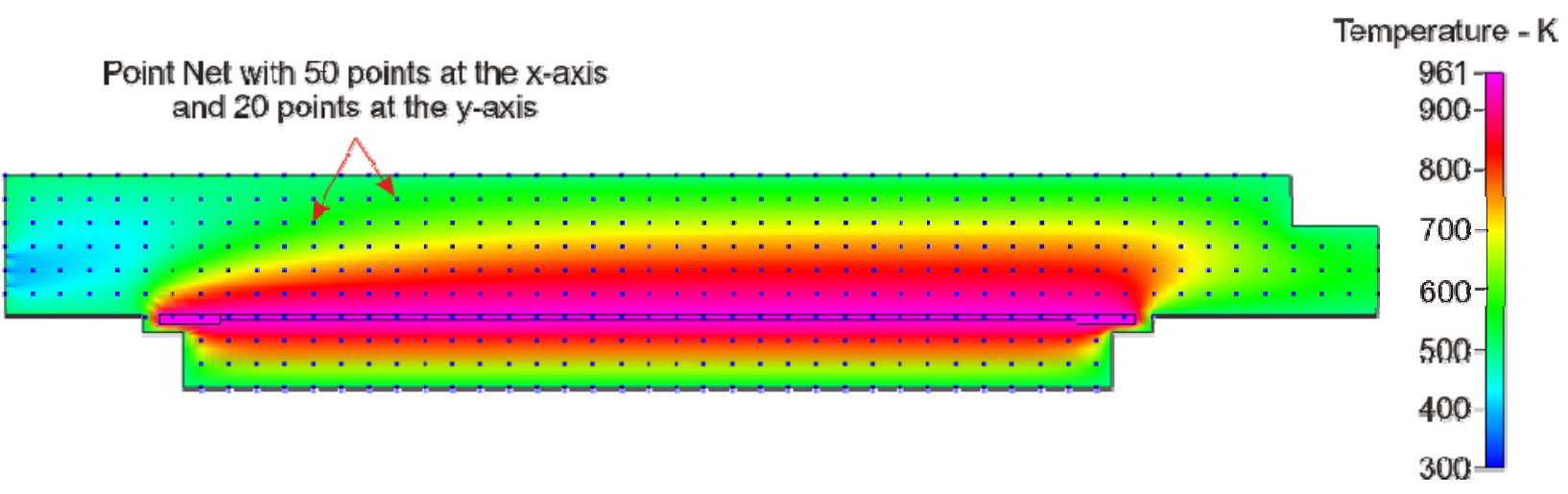

Figure 4: Simulation model of the RTP-reactor with colored temperature information inside the reaction chamber. The point net is used to read out the temperature information and simulate sensors (real point net resolution is higher).

\subsection{Description of the Graphical User Interface}

A Graphical User Interface (GUI) is set up for a comfortable user interaction. Figure 5 shows a screenshot of the GUI. Input data and VM algorithms can be selected; process and equipment behavior can be specified as described above. Finally, VM results are visualized and can be compared with each other and with measured values. The gage capability of the different VMs is displayed as well (Figure 6).

\subsection{Link of Statistical Methods with Physical Simulations}

After statistical changes to the historical data a physical simulation is executed for each wafer run. For each of these physical simulation runs the statistically changed values are added as boundary conditions to the CFD-ACE model. After the physical simulation is finished, the results of the physical simulation are extracted and interpreted as sensor values at specific points; see Figures 3 and 4 . These values are then added to the dataset in the Matlab/Simulink model. The Python scripting Application Programming Interface (API) of CFD-ACE is used to change the boundary conditions of the model and to get the sensor data from the simulation result at the desired points. Part of the results are interpreted as metrology results and used to judge the VM output later. The other part, serving as sensor data, is combined with the statistically altered fab data. Next, the VM algorithms are fed from the data pool with the input signals they need respectively.

At the end, the results of the VM algorithms are compared with each other and with metrology data from the simulation. Based on the difference their respective gage capability is calculated.

\section{ACHIEVED RESULTS}

Main results of the presented work are:

- The testing environment itself. To the knowledge of the authors, this is the first time a test bench for Virtual Metrology algorithms is developed and described. 


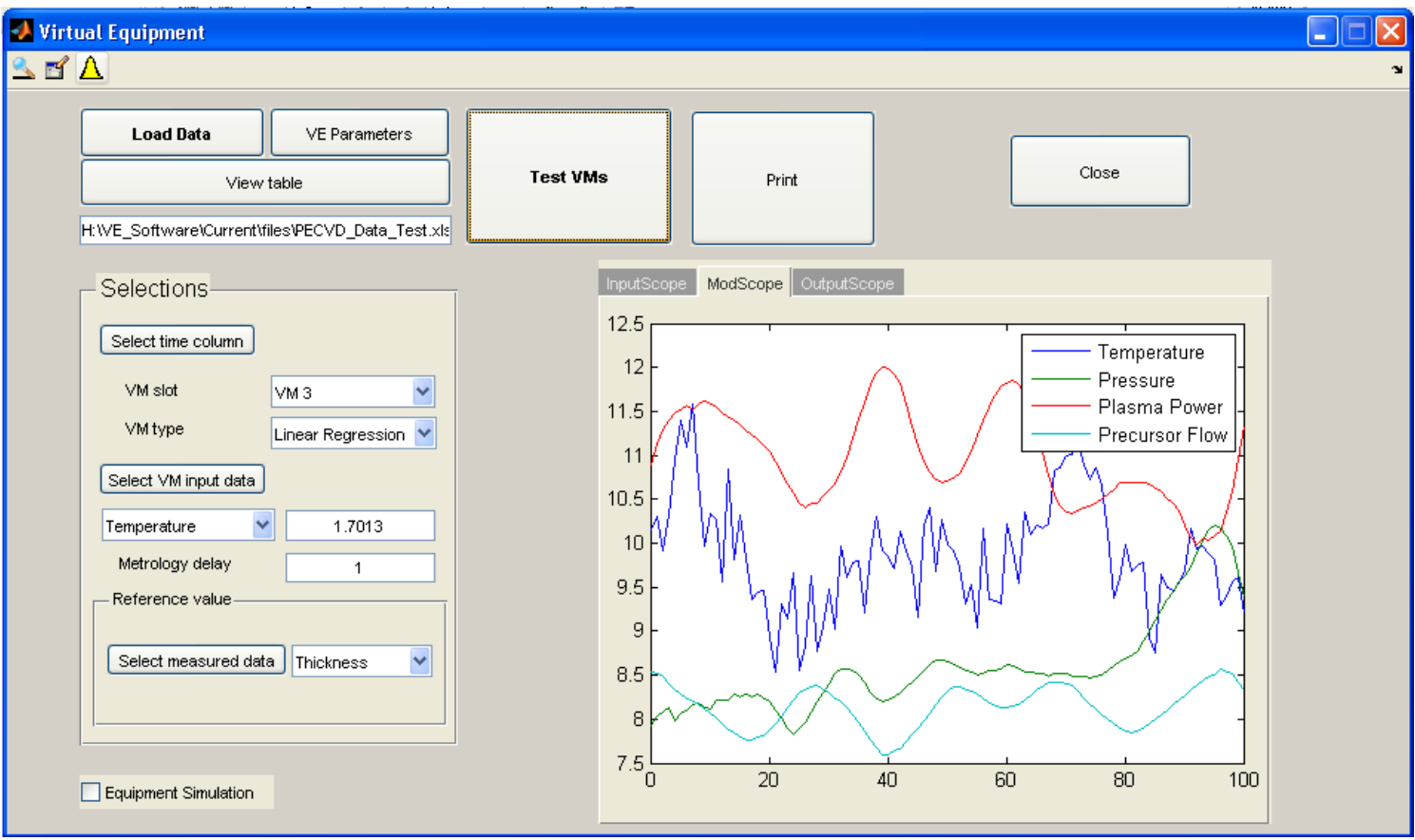

Figure 5: Screenshot of the Graphical User Interface

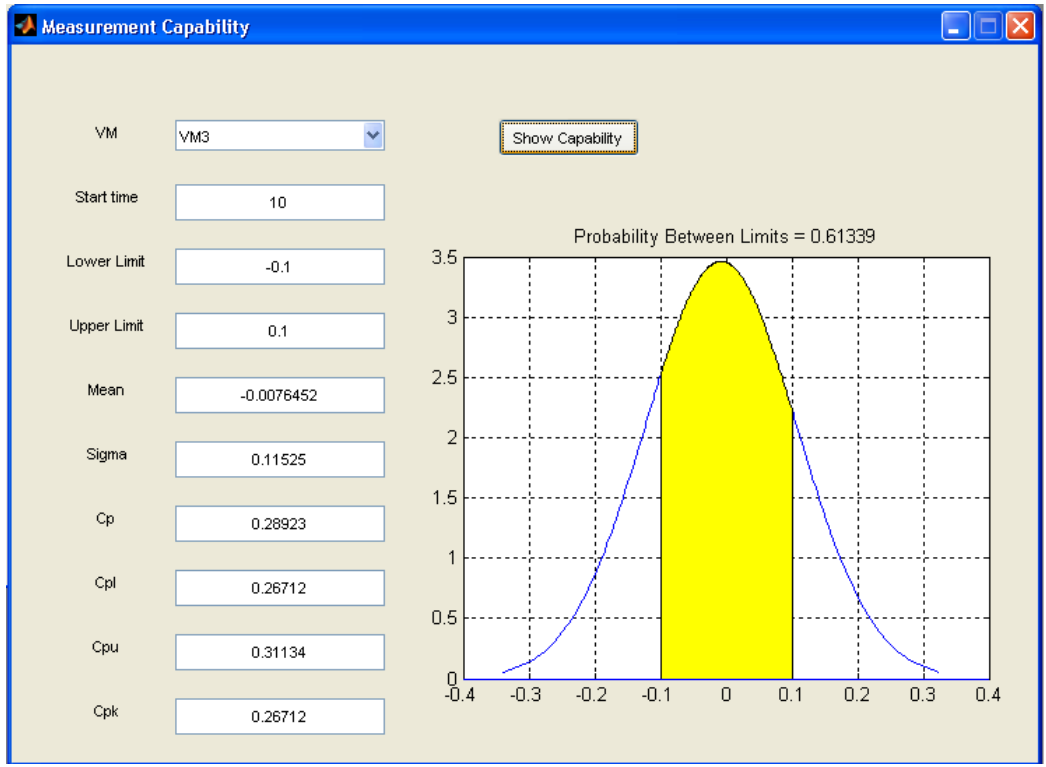

Figure 6: GUI showing the measurement capability of a Virtual Metrology model

- The Virtual Equipment provides a bidirectional link between statistical methods fed from actual historical fab data and physical simulations of a CVD reaction chamber.

- The test bench was applied to exemplary VM algorithms using linear regression algorithms and fixed linear approximation.

In this section, details about the application of the test bench to the exemplary VM algorithms are presented and discussed. The exemplary scenario was chosen as follows: 
- The virtual equipment to which the VM algorithms are applied uses a plasma based CVD process.

- Noise and offset simulate worse sensors in the equipment compared to the source data, both in terms of calibration and accuracy. This should have no direct effect on the process result so the VM algorithms need to compensate and the same metrology data as in the original data set can be used.

- The drifts added are supposed to be the result of changes to the reaction chamber as a side effect of the process. The process control of the equipment is assumed to counter these effects to keep the process result the same. So the original metrology data can be used.

- All three exemplary VMs start by applying a predefined fixed linear approximation. After sufficient metrology data is gathered, VM1 and VM2 switch to linear regression. The difference between them is the delay after which they have access to metrology data. VM1 gets metrology results later than VM2.

Figure 7 shows exemplary data which was used as input data for the virtual test bench. We used information about temperature, pressure, plasma power and precursor flow of the virtual reaction chamber. This input data was modified by applying noise, offsets and drifts as can be seen in Figure 8.

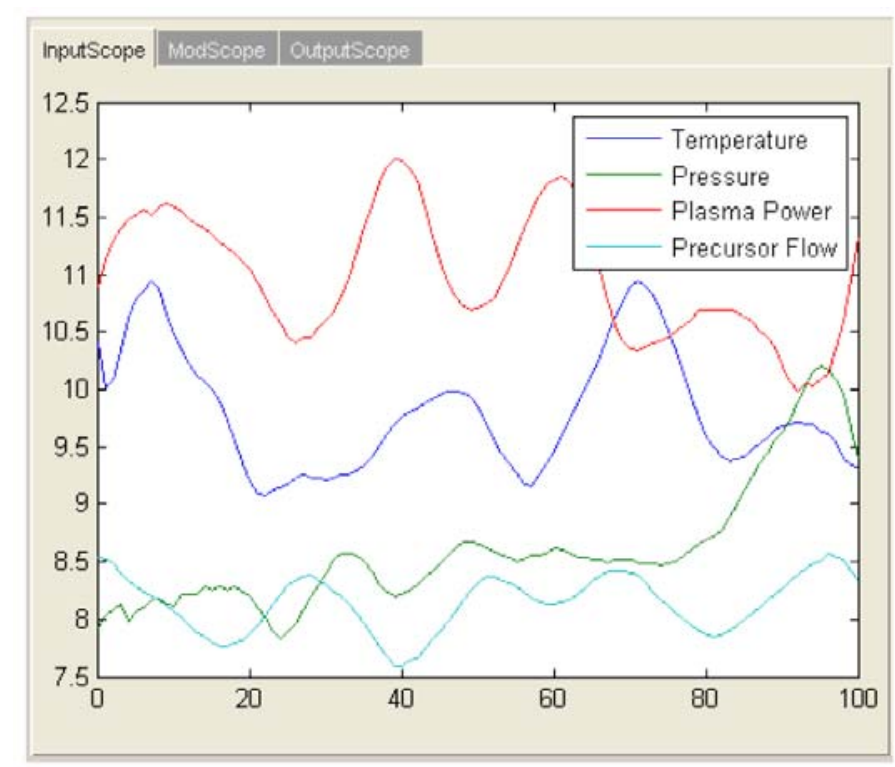

Figure 7: Exemplary input data, corresponds to green parts in Figures 1 and 2

The unchanged and modified data serve as two input data sets for the VM algorithms under investigation. Figure 9 depicts the results of the VM algorithms (fed by the original data set), compared to the real metrology data. Before sufficient metrology data is available, all three VMs use the same calculation method and the same parameterization. The time when VM1 and VM2 switch to linear regression is marked by sudden changes in the results. Due to the different metrology delays that happens earlier for VM2. Over the whole time period, VM3 shows the biggest deviations from the real metrology results whereas algorithms VM1 and VM2 predict the expected process result much better after a certain time.

Figure 10 shows the results of the VMs on the altered data. While all three algorithms can deal with the increased noise and offset, the fixed linear approximation VM cannot deal with the drift starting at time step 50. The linear regression VMs adapt to the drift but the effect of the metrology delay is stronger than without the drift. 


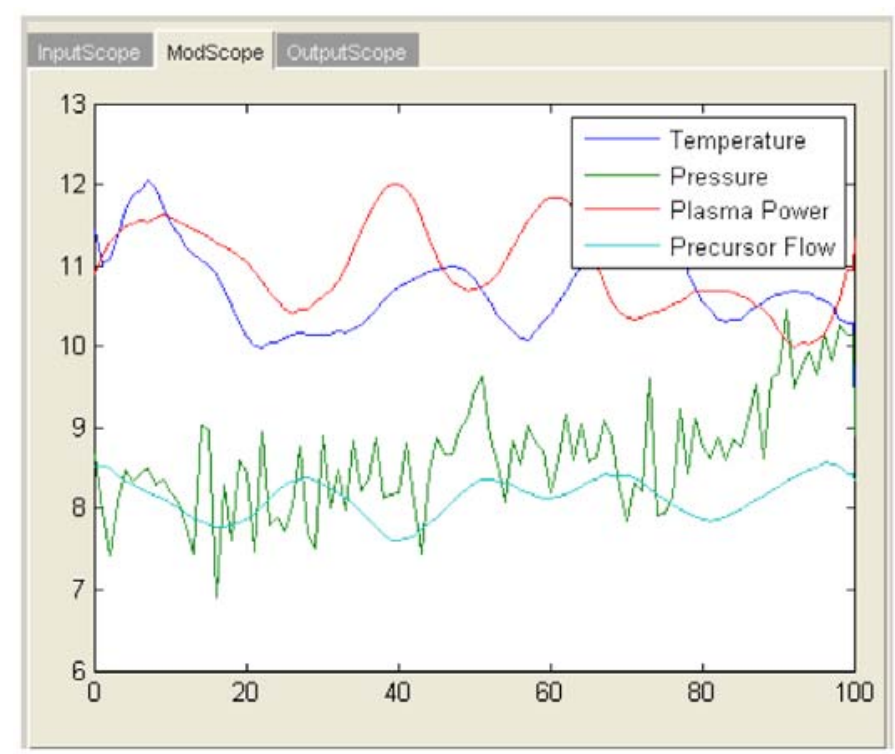

Figure 8: Modified data as input data for VM algorithms, corresponds to output of yellow part in Figures 1 and 2

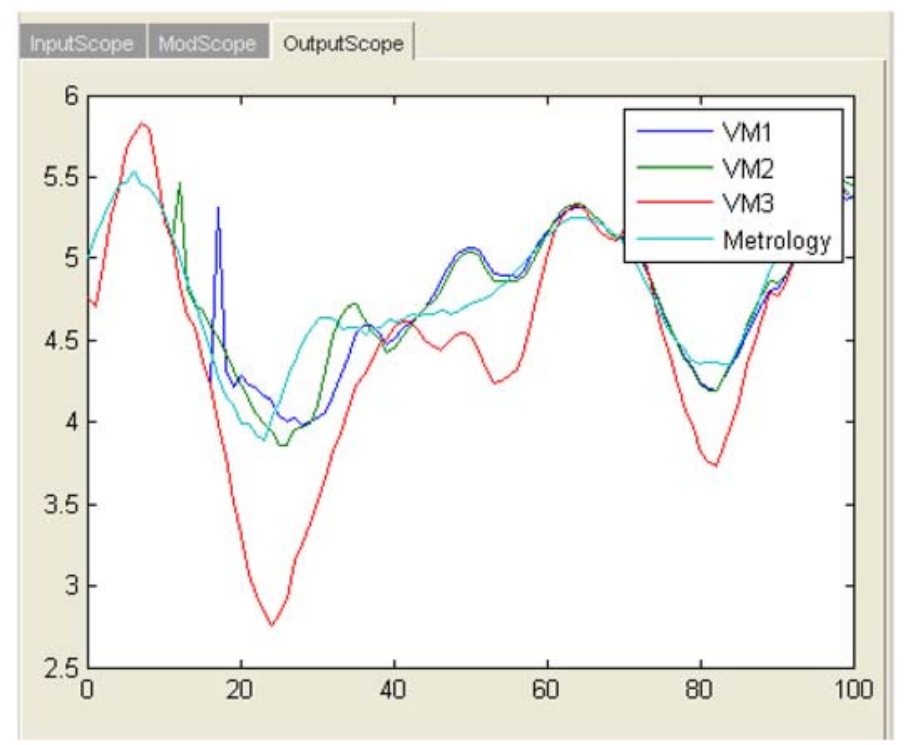

Figure 9: Comparison of output of VM algorithms on unaltered data and metrology data, corresponds to blue part in Figures 1 and 2

In this example, the two VM algorithms, VM1 and VM2, using linear regression algorithms predict the expected process results more accurately than VM3 which uses a fixed linear approximation.

\section{DISCUSSION AND NEXT STEPS}

The testing environment, presented in this paper, allows for testing and comparison of learning and static VM algorithms. In the future, the flexibility will be increased and it will be extended to include further automatic comparison studies. 


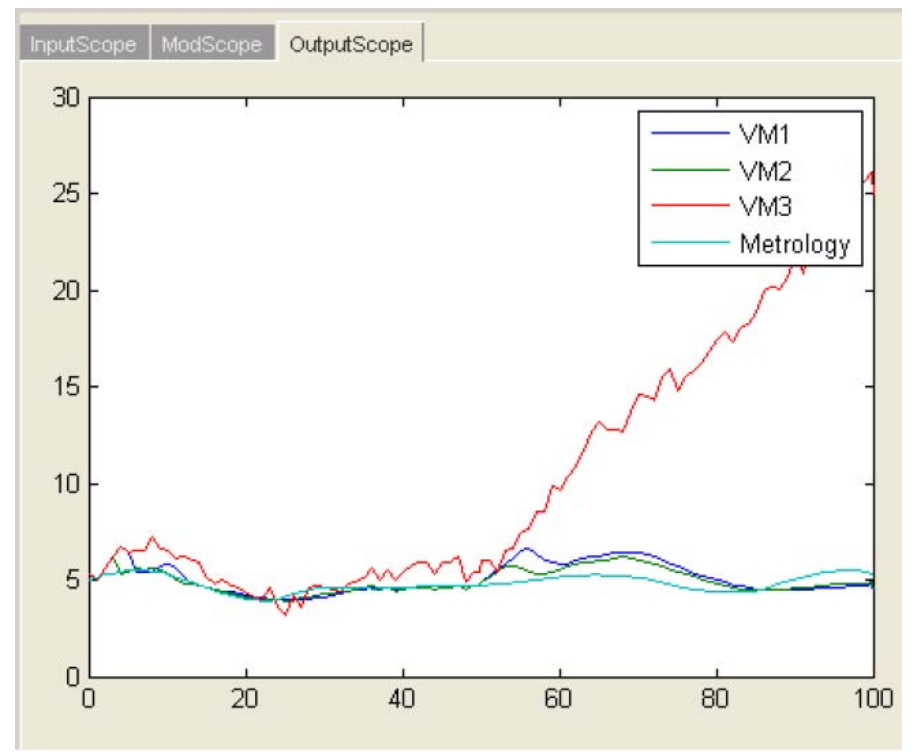

Figure 10: Comparison of output of VM algorithms on changed data and metrology data

The test bench takes into account historical fab data, synthetically generated data and data from physical simulations which serve as input data for testing different VM algorithms. Standard statistical methods are applied to modify the input data and to simulate various common and uncommon states of the process equipment. Especially critical scenarios which one would never test in real life can easily be evaluated without danger. Currently one test data set is generated. In the future, studies will be possible that execute multiple runs to evaluate a change parameter range or smoothen the random effect of nondeterministic methods. The next version of the test bench will take into account more precise equipment behavior during and after maintenance actions. That is why further tests are planned with IDM partners and equipment suppliers as well.

The presented test bench takes into account the physical simulation of a CVD reaction chamber. VM algorithms will be applied to different types of equipment, e.g., plasma etchers, furnaces, etc. Future version of the test bench will integrate simulations of different equipment types. In addition, as an extended statistical method, Bayesian Networks will be used to learn the interdependence of the signals to keep the behavior of signals consistent after changes to single signals.

\section{SUMMARY AND CONCLUSION}

Currently, a variety of new VM algorithms for different processes are being developed. Prior to their implementation into the IT structure of IDMs' factories, novel VM algorithms have to be tested. This paper presented a testing environment for evaluating VM algorithms. The test bench merges statistical simulation with physical simulation. It generates test data sets for various common and uncommon states of the processing equipment. Data basis are historical fab data, synthetically generated data and data from physical simulations.

Main results of our work are the bidirectional link between statistical methods fed from actual history fab data and physical simulations, set up of the virtual testing environment, and a GUI for evaluating VM algorithms and the set-up of a simplified physical simulation of a CVD reaction chamber based on CAD data.

Future work will include the following steps:

- Verification of simulated process behavior with real data from project partners

- Evaluation of VM algorithms provided by project partners

- Further equipment simulation, e.g. plasma etch, furnaces, etc. 
Mattes, Koitzsch, Lewke, Müller-Zell and Schellenberger

\section{ACKNOWLEDGEMENTS}

This work was in parts funded by the EU-project IMPROVE, contract no. 120005.

\section{REFERENCES}

Chang, Y.-J., Y. Kang, C.-L. Hsu, C.-T. Chang, and T. Y. Chan. 2006. "Virtual Metrology Technique for Semiconductor Manufacturing". In Proceedings of the International Joint Conference on Neural Networks, 5289-5293. Vancouver, BC, Canada.

ESI-Group. 2006. "Simulation of Polysilicon Deposition in a Jipelec RTP Reactor Using CFD-ACE+." CFD-ACE+ Tutorial.

Fair, R.B. 1993. Rapid Thermal Processing: Science and Technology. Boston, San Diego, New York, London, Sydney, Tokyo, Toronto: Academic Press.

Hung, M. H., T. H. Lin, P. H. Chen, and R. C. Lin. 2007. "A Novel Virtual Metrology Scheme for Predicting CVD Thickness in Semiconductor Manufacturing." IEEE/ASME Transactions on Mechatronics 12(3):308-316.

Kim, S. J., and Y. M. Cho. 2002. "Optimal Design of a Rapid Thermal Processor Via Physics-Based Modeling and Convex Optimization." Control Engineering Practice 10:1199-1210.

Lewke, D. 2011. "Gerätesimulation basierend auf einer parametrisierten RTP-Reaktorgeometrie und Validierung ausgewählter Parameter." Diploma thesis. Friedrich-Alexander University of ErlangenNuremberg.

Moore, G. E. 1965. “Cramming More Components onto Integrated Circuits.” Electronics 38(8):114-117.

\section{AUTHOR BIOGRAPHIES}

ANDREAS MATTES is a research scientist at Fraunhofer IISB. He holds a diploma in computer science from the University of Stuttgart and joined Fraunhofer IISB in 2007. Andreas Mattes is working on the topic of equipment and process simulations for semiconductor manufacturing equipment. E-mail: andreas.mattes@iisb.fraunhofer.de

MATTHIAS KOITZSCH is a research scientist with Fraunhofer IISB since 2006. He is active in the areas of technology development for the microelectronics industry and return on investment calculations. He holds a diploma in electrical engineering from the Friedrich-Alexander University of ErlangenNuremberg. E-mail: matthias.koitzsch@iisb.fraunhofer.de

DIRK LEWKE is a research scientist at Fraunhofer IISB. He holds a diploma in mechatronics from the Friedrich-Alexander University of Erlangen-Nuremberg. Dirk Lewke is active in the field of integrated metrology and equipment simulation for the semiconductor industry. E-mail: dirk.lewke@iisb.fraunhofer.de

MICHAEL MÜLLER-ZELL is a graduate student of mathematics at the University of Applied Sciences Regensburg. At Fraunhofer IISB, he was active in the field of software development for test environments regarding novel Virtual Metrology algorithms. E-mail: michael.mueller-zell@iisb.fraunhofer.de

MARTIN SCHELLENBERGER received his diploma in Electrical Engineering in 1998 from the Friedrich-Alexander University of Erlangen-Nuremberg, Germany. As group manager for equipment and advanced process control at Fraunhofer IISB, he is responsible for the development of solutions for integrated metrology and sensors and their link to advanced process control systems. E-mail: martin.schellenberger@iisb.fraunhofer.de 